\title{
Voltage Stability of Multiple Distributed Generators in Distribution Networks
}

\author{
Andi Wang ${ }^{1,2}$, Chongxin Liu ${ }^{1, *}$, Hervé Guéguen ${ }^{2}$ and Zhenquan Sun ${ }^{3}$ \\ ${ }^{1}$ School of Electrical Engineering, Xi'an Jiaotong University, Xi'an 710049, China \\ ${ }^{2}$ CentraleSupélec-IETR, Avenue de la Boulaie, CS47601, F-35576 Cesson-Sévigné Cedex, France \\ ${ }^{3}$ Shaanxi Regional Electric Power Group Co., Ltd. Qindian International, Xi'an 710061, China \\ ${ }^{*}$ Corresponding author
}

\begin{abstract}
Recent research has proved that, the local reactive power control laws considered to solve voltage stability issue might lead to voltage instability. For this reason, the current paper proposes an approach to study voltage stability of multiple distributed generators in distribution feeders. The methodology relies on the construction of an equivalent state space model of the continuous system. A simulation based on MATLAB of the proposed approach to a real medium-voltage 26 buses network with 3 distributed generators reveals unstable operating situations of the network and helps to design a set of measurement filters to ensure system stability.
\end{abstract}

Keywords-distributed generators; voltage stability; measurement filter

\section{INTRODUCTION}

Over the past decade, due to the dramatic increase of Distributed Generators (denoted as DG), the power flows of distribution networks have been substantially modified. Several solutions have been investigated to be able to maintain the voltage within specified limits while avoiding network reinforcement costs [1] [3] [4]. Among these ideas, local control laws of DG reactive power (Q) as a function of their voltage $(\mathrm{U})$ have been highly considered.

In this paper, a methodology is developed to study voltage stability of distribution feeders with $Q(U)$ laws. Due to the nonlinearity of the $\mathrm{Q}(\mathrm{U})$ law, classical stability analysis cannot be used here. To assess system stability, the system is modeled as a continuous transition system using hybrid system tools [2]. Because of the infinite number of possible operating voltages, unsafe regions are still difficult to study. Starting from a continuous system, the authors of [2] propose an equivalent finite discrete transition system which simplifies the analysis of safe operations. The aim of this paper is to use discrete abstraction techniques to construct a discrete state space equivalent to the continuous model of a medium-voltage (MV) feeder connecting multiple distributed generators. The main contribution consists of developing a method to conduct a formal stability analysis of the system allowing to set measurement filter and $\mathrm{Q}(\mathrm{U})$ law parameters in order to ensure voltage stability.

In a distribution grid, distributed generators can be used to regulate voltage profile. With the help of measurement filters and $\mathrm{Q}(\mathrm{U})$ regulators, producers adjust their reactive power outputs to restore the voltage magnitude to its nominal value.

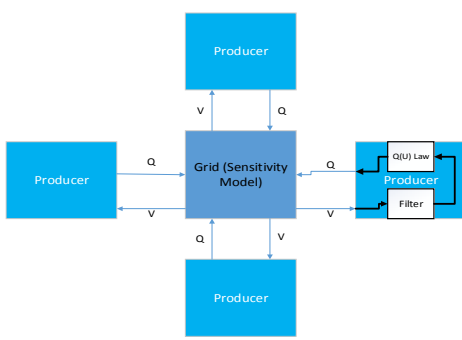

FIGURE I. STABILIZING THE GRID VOLTAGE PROFILE WITH THE HELP OF DISTRIBUTED PRODUCERS

First of all, the system is presented in Section II. It details the construction of the equivalent discrete system. In Section III, system stability is discussed and a measurement filter ensuring system stability is proposed. Robustness is analyzed in Section IV. A real case simulation is presented in Section V. Conclusions and perspectives are presented in Section VI.

\section{THEORETICAL MODELING}

\section{A. State Space Model}

When introducing the $\mathrm{Q}(\mathrm{U})$ law to rebalance the voltage magnitude, instability might happen. And we can do little to improve it, due to the determined feedback system structure.

That's why we adopt a filter (e.g. a first order autoregressive filter) which can give us more flexibility and possibility in regulating the voltage profile further (shown in Figure 2,

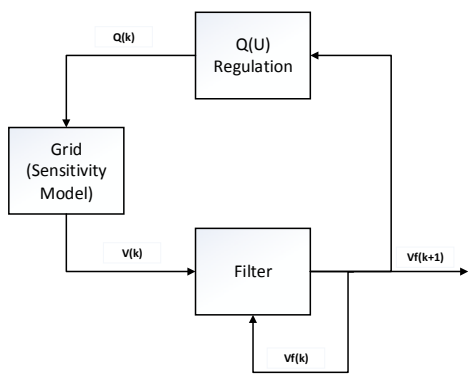

FIGURE II. CONTROL STRUCTURE

A first order autoregressive filter can be expressed as:

$$
V_{\text {filt }}(k+1)=A \cdot V_{\text {filt }}(k)+(\mathrm{I}-A) \cdot V(k)
$$


In which, $V_{\text {filt }}(k)$ is a vector representing the outputs of filters in each bus, while $\mathrm{V}(\mathrm{k})$ is the voltage magnitudes regarded as inputs.

$$
A=\left[\begin{array}{lll}
a_{1} & & \\
& \ddots & \\
& & a_{n}
\end{array}\right]
$$

$a_{i}(i=1, \ldots, n)$ represents the parameter of the $i$-th filter.

Now we can establish a state space, considering $\mathrm{V}_{\text {filt }}(\mathrm{k})$ as state variables.

For simplification reason, we apply sensitivity analysis to the grid. So that we only focus on how reactive power injection impacts on the voltage magnitude, which refers to $\partial \mathrm{V} / \partial \mathrm{Q}$. When the voltage magnitude (V) is around its nominal value, it will be almost aligned with reactive power. We assume the voltages are around nominal values, which is reasonable, then we can model the grid as:

$$
V(k)=K \cdot Q(k)+V_{0}
$$

In which $\mathrm{K}$ is a matrix, whose entries representing the values of $\partial \mathrm{V} / \partial \mathrm{Q}$ when $\mathrm{V}$ equals to its nominal value.

Q (U) law refers to the regulation command sent to the producer (shown in Figure 3).

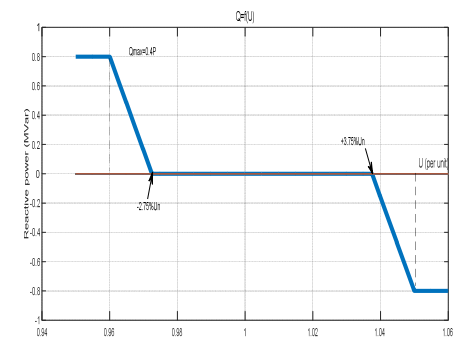

FIGURE III. A TYPICAL REPRESENTATION OF Q (U) LAW

It's a piecewise function with 5 states which can be classified into 2 kinds, whether $\mathrm{Q}$ is constant, or Q is linear with $\mathrm{V}_{\text {filt. }}$

We observe that, in the 2 sections of linear parts, the slopes are the same. Let's note the slope as $c_{i}$ for i-th producer.

So we can always have the equations:

$$
Q(k)=C \cdot V_{f i l t}(k)+Q_{0}
$$

With the entries of matrix $\mathrm{C}$ :

$$
\left\{\begin{array}{c}
c_{i}=0\left(\text { when } Q_{\text {inj }} \text { is in constant state }\right) \\
c_{i} \neq 0\left(\text { when } Q_{\text {inj }} \text { is linear with } V_{\text {filt }}\right)
\end{array}\right.
$$

Now we substitute (3) (4) into (1), which will be:

$$
\begin{aligned}
V_{f i l t}(k+1)= & (A+(\mathrm{I}-A) \cdot K \cdot C) V_{f i l t}(k)+(\mathrm{I}-A) \cdot K \cdot C \\
& \cdot Q_{0}+V_{0}
\end{aligned}
$$

So the necessary and sufficient condition of system stability is that, all the eigenvalues of $\mathrm{A}+(\mathrm{I}-\mathrm{A}) \cdot \mathrm{K} \cdot \mathrm{C}$ are in unit circle.
Firstly, suppose we only have 2 DGs.

$$
=\left[\begin{array}{cc}
A+(\mathrm{I}-A) \cdot K \cdot C \\
a_{1}+\left(1-a_{1}\right) k_{11} c_{1} & \left(1-a_{1}\right) k_{12} c_{2} \\
\left(1-a_{2}\right) k_{21} c_{1} & a_{2}+\left(1-a_{2}\right) k_{22} c_{2}
\end{array}\right]
$$

\section{StABILITY ANALYSIS}

Depending on the value of $\mathrm{c}_{\mathrm{i}}$, there are three cases.

Case 1: $c_{1}=c_{2}=0$

In this case,

$$
A+(\mathrm{I}-A) \cdot K \cdot C=\left[\begin{array}{ll}
a_{1} & \\
& a_{2}
\end{array}\right] .
$$

Which means stability is always accomplished since $0<$ $a_{i}<1$.

Case 2: one of $c_{i}$ equals 0 , while the other doesn't

For example, $c_{1}=0, c_{2} \neq 0$

$$
\begin{gathered}
A+(\mathrm{I}-A) \cdot K \cdot C=\left[\begin{array}{cc}
a_{1} & \left(1-a_{1}\right) k_{12} c_{2} \\
0 & a_{2}+\left(1-a_{2}\right) k_{22} c_{2}
\end{array}\right] \\
-1<a_{2}+\left(1-a_{2}\right) k_{22} c_{2}<1
\end{gathered}
$$

This is the stability criterion when there is only 1 DG. It shows that, one necessary condition of stability in an n-DGs system is, each combination of its n-1 DGs are set to work stably in the case without the other one DG.

Matrix A has to satisfy:

$$
a_{i}>\frac{-1-k_{i i} c_{i}}{1-k_{i i} c_{i}}
$$

Case 3: neither of them equals 0

$$
\begin{aligned}
& A+(\mathrm{I}-A) \cdot K \cdot C \\
& =\left[\begin{array}{cc}
a_{1}+\left(1-a_{1}\right) k_{11} c_{1} & \left(1-a_{1}\right) k_{12} c_{2} \\
\left(1-a_{2}\right) k_{21} c_{1} & a_{2}+\left(1-a_{2}\right) k_{22} c_{2}
\end{array}\right.
\end{aligned}
$$

For simplification, let's note it as $\left[\begin{array}{ll}X & W \\ Z & Y\end{array}\right]$, with $\mathrm{X}, \mathrm{Y}, \mathrm{Z}$ and $\mathrm{W}$ equal to the entries respectively.

The 2 eigenvalues of this matrix are:

$$
\rho_{1,2}=\frac{X+Y \pm \sqrt{(X+Y)^{2}-4(X Y-W Z)}}{2}
$$

We choose the range of $a_{1}$ so that $-1 / 2<\mathrm{X}<0$, as well as such $a_{2}$ that $-1 / 2<\mathrm{Y}<0$. Then $-1<\mathrm{X}+\mathrm{Y}<0$.

$$
\sqrt{(X+Y)^{2}-4(X Y-W Z)}=\sqrt{(X-Y)^{2}+4 W Z}
$$

In which

$$
-1 / 2<\mathrm{X}-\mathrm{Y}<1 / 2 \text {, so } 0<(X-Y)^{2}<\frac{1}{4} \text {. }
$$

As long as $\sqrt{(X+Y)^{2}-4(X Y-W Z)}<1$, we'll get $-2<$

$$
X+Y \pm \sqrt{(X+Y)^{2}-4(X Y-W Z)}<1
$$

Which means,

$$
-1<\frac{X+Y \pm \sqrt{(X+Y)^{2}-4(X Y-W Z)}}{2}<\frac{1}{2}
$$

It will be within the unit circle.

In order to make

$$
\begin{aligned}
& \sqrt{(X+Y)^{2}-4(X Y-W Z)}=\sqrt{(X-Y)^{2}+4 W Z}<1 \\
& \text { Choose the value of } a_{1}, a_{2}, \text { so that }
\end{aligned}
$$




$$
\mathrm{W}, \mathrm{Z}>-\frac{\sqrt{3}}{4}
$$

Conclude the inequations above, we will get the range of A.

In conclusion,

$$
\left\{\begin{array}{c}
a_{i}>\frac{-\frac{1}{2}-k_{i i} c_{i}}{1-k_{i i} c_{i}} \\
a_{i}>1-\frac{\sqrt{3} / 4}{k_{i j} c_{j}}
\end{array}\right.
$$

We found that, these criteria is sufficient to satisfy the criterion for a 1-DG system, Inequation (5). We conclude, for a system with $\mathrm{N}$ DGs, the most possibly unstable case is that every single DG is in the linear section of Q (U) law.

So we can also deduce that, if a system is able to keep stable when every single bus is in a linear section of Q (U) law, then this system is stable in all the cases.

All the procedures above achieve to find such a resolution that stabilize a distribution grid only with 2 DGs. Nevertheless, we could prove that, these criteria are also a set of necessary conditions for a system with 3 or more DGs.

For example, in a 3-DG distribution system, the criterial matrix now becomes 3-by-3:

$$
\begin{aligned}
& (A+(\mathrm{I}-A) \cdot K \cdot C)= \\
& {\left[\begin{array}{ccc}
a_{1}+\left(1-a_{1}\right) k_{11} c_{1} & \left(1-a_{1}\right) k_{12} c_{2} & \left(1-a_{1}\right) k_{13} c_{3} \\
\left(1-a_{2}\right) k_{21} c_{1} & a_{2}+\left(1-a_{2}\right) k_{22} c_{2} & \left(1-a_{2}\right) k_{23} c_{3} \\
\left(1-a_{3}\right) k_{31} c_{1} & \left(1-a_{3}\right) k_{32} c_{2} & a_{3}+\left(1-a_{3}\right) k_{33} c_{3}
\end{array}\right]}
\end{aligned}
$$

With my knowledge, it's very hard to find a set of appropriate values for $a_{i}$ so that all the eigenvalues will be within the unit circle.

However, let's imagine in case that the third DG comes to a state that the voltage is around nominal value, then reactive power injection drops to the dead zone. That is to say $c_{3}=0$.

Then the criteria matrix becomes

$$
=\left[\begin{array}{ccc}
(A+(\mathrm{I}-A) \cdot K \cdot C) \\
a_{1}+\left(1-a_{1}\right) k_{11} c_{1} & \left(1-a_{1}\right) k_{12} c_{2} & 0 \\
\left(1-a_{2}\right) k_{21} c_{1} & a_{2}+\left(1-a_{2}\right) k_{22} c_{2} & 0 \\
\left(1-a_{3}\right) k_{31} c_{1} & \left(1-a_{3}\right) k_{32} c_{2} & 0
\end{array}\right]
$$

Calculating the eigenvalues of this matrix will be exactly the same as what we did in the case of 2-DG system. That is to say, each two of the 3 DGs running stably is a prerequisite of the stability of a 3-DG system. So we can still apply our method in this case, to get an answer which is very close to the optimal solution. We can verify this hypothesis in the simulation.

\section{ROBUSTNESS ANALYSIS}

Another point that I want to address is how network reconfiguration impacts on the regulation. That is to say, how is the robustness?
Among all the parameters in our conclusions, except for grid sensitivity model, nothing would change when reconfigured. Let's see how sensitivity model will change.

Assume node $\mathrm{X}$ is one of the buses in a system. After applying nodal analysis on it, we get:

$$
I_{\text {from other nodes }}=I_{\text {net }} \text { power }-I_{\text {capacitance }}
$$

If there are $\mathrm{N}$ buses connected to node $\mathrm{X}$,

$$
\frac{V_{1}-V_{x}}{z_{1 x}}+\cdots+\frac{V_{N}-V_{x}}{z_{N x}}=\frac{P_{x}-j Q_{x}}{V_{x}}-\frac{1}{2}\left(b_{1 x}+\cdots+b_{N x}\right) V_{x}
$$

$z_{1 x}$ refers to the impedance between Bus 1 and node $\mathrm{X}$, $b_{1 x}$ refers to the admittance between Bus 1 and node $\mathrm{X}$.

If we derivate it, we will get:

$$
\frac{\partial V_{x}}{\partial Q_{x}}=\frac{1}{\frac{V_{1}-2 V_{x}}{z_{1 x}}+\cdots+\frac{V_{N}-2 V_{x}}{z_{N x}}+\left(b_{1 x}+\cdots+b_{N x}\right) V_{x}}
$$

Which indicates that, the self-sensitivity model of a node is influenced merely by the line parameters between the node and other nearest nodes, as well as their voltage magnitudes.

We can also deduce,

$$
\frac{\partial V_{1}}{\partial Q_{x}}=\frac{z_{1 x}}{V_{x}}
$$

This suggests the mutual sensitivity model is influenced merely by the impedance between the two nodes as well as the generator voltage magnitude.

Generally speaking, the diagonal entries of sensitivity matrix are only affected by the network structure linking to the DG nodes. If we reconfigure the nodes which are far from the DG buses, then it will not impact much on the sensitivity model.

I think we can neglect the variations of voltage profile, since it's always close to nominal value. That is to say, the nondiagonal entries of sensitivity model can be regarded as unchanged when network reconfigured.

\section{EXPERIMENTAL VALIDATION}

I built a 26-bus distribution system as a test case (shown in Figure 4). The nominal voltage amplitude value is $20 \mathrm{kV}$. The system consists of 2 subsystems. Buses of 1 to 13 make up Subsystem 1, with a distributed generator connected to Bus 3, while Buses of 14 to 26 make up Subsystem 2, with 2 distributed generators connected to Bus 18 and 20 respectively.
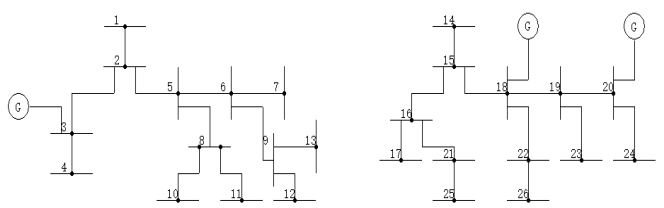

FIGURE IV. THE ORIGINAL NETWORK STRUCTURE

The left subsystem is heavy-loaded while the right one produce too much active power. So the grid operator wants to make a change to the topological structure of the network (shown in Figure 5). 


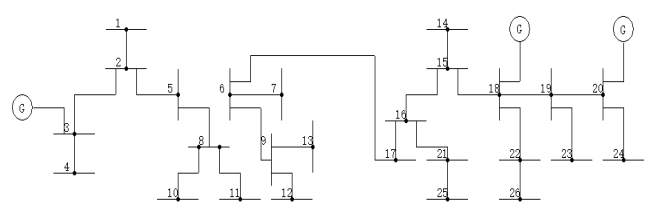

FIGURE V. THE NEW NETWORK STRUCTURE

It helps rebalance the power consumption and production. Nevertheless, this variation might produce overvoltage. For this reason, we introduce our regulation method.

The system is assumed to consist of 4 states.

State 1 is just like the initial state with all the data initialization. In State 2, we keep the network structure, but increase the power demanding in bus 12 from 1 to $2 \mathrm{MW}$. In State 3, we switch to the new network structure in Figure, everything else remains. In State 4, we restore the power demanding in bus 12 to its original value.

We simulate the 4 states successively, keeping an eye on the voltage profile of the 3 buses with DGs.

Figure 6 shows the voltage profile of all the 3 DG buses without the help of measurement filters. The instability occurs obviously.
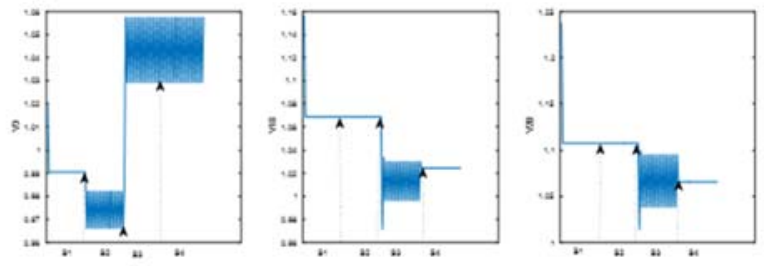

FIGURE VI. THE VOLTAGE PROFILE OF THE DG BUSES (BEFORE)

According to the analysis above, we regard the model as constant during the state transition. So firstly I got the sensitivity model of the initial state in a numerical way, which determined the $\mathrm{K}$ matrix. Then we can determine the $\mathrm{C}$ matrix from Q (U) law, which is constant as well. In the end, we can apply the inequations (6) to determine the value of matrix A, which will be the filter parameters.

Finally, we run the simulation with parameters set correctly, and observe the results (Figure 7). Comparing to the previous results, the voltage profile keeps much more stable. And even if the system structure undergoes a set of big changes, the voltage profile is stable and transits smoothly overall.
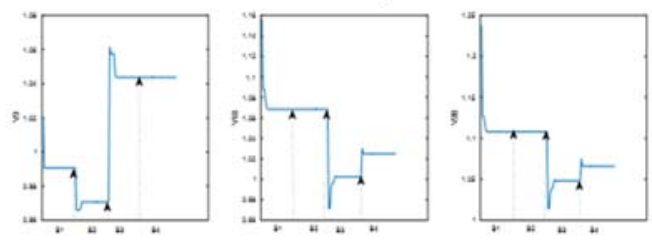

FIGURE VII. THE VOLTAGE PROFILE OF THE DG BUSES (AFTER)

\section{CONCLUSION}

The proposed methodology relies on the construction of an equivalent discrete state space model to study the stability of the nonlinear system. Then an experimental validation allows to conclude on system stability. This method is illustrated on a real 26 nodes distribution feeder hosting three DGs. The case study seems stable after a rapid empirical study. A set of measurement filters are added to stabilize the system. The proposed method is also used to choose a filter ensuring system stability.

In the future, I will dig into the case with 3 or more DGs. Certainly, as DGs multiply on feeders, instability issues will increase.

\section{REFERENCES}

[1] A. Allen, Y. Zhang, and B. Hodge, Voltage Impacts of Utility-Scale Distributed Wind. National Renewable Energy Laboratory, 2014.

[2] R. Alur, T. A. Henzinger, G. Lafferriere, and G. J. Pappas, "Discrete abstractions of hybrid systems," Proc. of the IEEE, vol. 88, no. 7, 2000.

[3] J. H. Braslavsky, J. K. Ward and L. Collins, "A stability vulnerability in the interaction between Volt-VAR and Volt-Watt response functions for smart inverters" in 2015 IEEE Conference on Control Applications, 2015.

[4] M. Cosson, H. Guéguen, D. Dumur, C. S. Maniu, V. Gabrion and G. Malarange, "Voltage Stability of Distributed Generators by means of Discrete Abstraction" in 2015 IEEE Conference on Control Applications, 2015 